\title{
ZUR GESCHICHTE DER SCHWEIZERISCHEN LANDESVERMESSUNG
}

\author{
VOn ED. IMHOF
}

Mit 1 Karte

Die Schweiz besaß bisher zwei grundlegende Quellenwerke ihrer Vermessungs- und Kartengeschichte: die 1878 erschienene "Geschichte der Vermessungen in der Schweiz» von Prof. Dr. RuDOLF WOLF und das aus jüngster Zeit stammende inhaltsreiche Buch von Prof. Dr. Leo Weisz über "Die Schweiz in alten Karten».

Hans Zoelly, Ehrendoktor der Eidgenössischen Technischen Hochschule und einstiger Chefstellvertreter der Eidgenössischen Landestopographie, fügt nun ein drittes Werk von fundamentaler

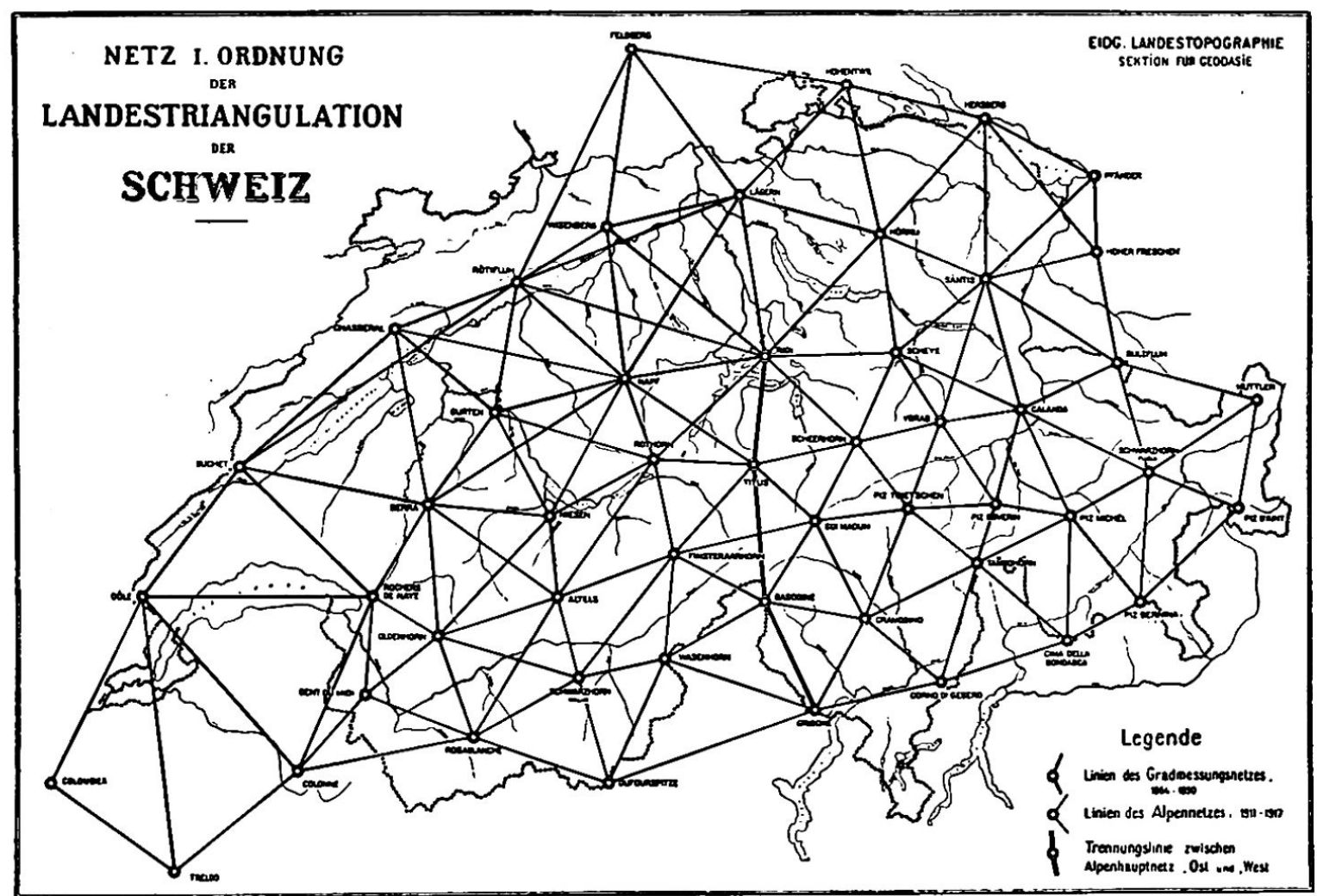

Abbildungsbeispiel aus H. Zoelly: Geschichte der geodätischen Grundlagen für Karten und Vermessungen in der Schweiz (Abb. 91), ca. 1: 3000000. Das trigonometrische Netz I. Ordnung

Bedeutung bei ${ }^{1}$. Er krönt damit seine eigene Lebensarbeit. Als langjähriger Leiter der geodätischen Arbeiten unserer Landesvermessung konnte er, wie kein anderer, aus einem reichen Schatz persönlicher Erfahrungen und Erinnerungen schöpfen. Sein Buch behandelt in unantastbarer Zuverlässigkeit und meisterhafter Formulierung die trigonometrischen Arbeiten vor 1785 und diejenigen der Übergangsperiode 1785-1830, die geodätischen Grundlagen für die Dufourkarte (1809-1864), die Arbeiten der Schweizerischen Geodätischen Kommission, die geodätischen Arbeiten für den Siegfriedatlas, für Kataster- und Forstvermessungen usw. und diejenigen im 20. Jahrhundert mit Einschluß der Messungen von Terrainbewegungen, von Staumauerdeformationen usw. Die einzigartige Dichte und Qualität des geodätischen Fixpunktnetzes der Schweiz, die besonderen Schwierigkeiten des alpinen Geländes, die führende Stellung des schweizerischen Instrumentenbaues und manche durch unsere Geodäten ein-

I Zoelly, HaNs: Geschichte der geodätischen Grundlagen für Karten und Vermessungen in der Schweiz. Verlag der Eidgenössischen Landestopographie in Wabern bei Bern. 160 Seiten (Folioformat), 129 Abbildungen und eine Netzkarte als Beilage. Preis Fr. 15.- 
gefuihrte Neuerung geben dem Buche von HaNs Zoelly große Bedeutung und machen es zu einem Denkmal schweizerischer Leistung.

Die Eidgenössische Landestopographie scheute keine Mühe, das Werk auch drucktechnisch und durch ausgezeichnete Abbildungen würdig auszustatten.

Dringend erwünscht wäre es, wenn uns in absehbarer Zeit und bevor alle Fäden persönlicher Erinnerung an die hohe Zeit der Siegfriedkarte abgerissen sein werden, auch eine gleichwertige Darstellung der neueren staatlichen Topographie und Kartographie geschenkt würde.

\section{NEUIGKEITEN - NOVA}

Verlagerungen in der Welterdölwirtschaft. Im Jahre 1938 verbrauchten die sog. CEEC.-Länder (Committee of European Economic Cooperation) 36224000 Tonnen Erdöl und Erdölderivate; nur Deutschland wies unter diesen Ländern eine bedeutendere Eigenproduktion (552000 Tonnen) auf, so da $\beta$ der Importbedarf mit etwas über 35 Millionen Tonnen angesetzt werden kann. Rund $40 \%$ dieses Bedarfes entfielen auf gewöhnliches Benzin; davon gingen $30 \%$ nach dem Vereinigten Königreich, $21 \%$ nach Frankreich, $8 \%$ nach Italien. Frankreich und in kleinerem Maße Italien waren bedeutend als Importeure von rohem Erdöl, das sie in landeseigenen Raffinerien verarbeiteten; die andern Länder importierten in erster Linie Erdölderivate. Als wichtigstes Bezugsgebiet galt das karibische Amerika und die USA. (Golfgebiet), die zusammen rund $40 \%$ der Benzinimporte deckten. Iran war nur mit $9 \%$ beteiligt. Die irakische Produktion erreichte als rohes Erdöl vor allem den französischen Markt. Außerhalb der USA. war Europa weitaus der wichtigste Markt für Erdöl und seine Derivate.

Die Nachkriegszeit hat hier in wesentlichen Punkten Wandlungen gebracht. Zahlreiche europäische Länder (vor allem Großbritannien und die Niederlande) erschlossen landeseigene Erdölfelder; viele entwickelten eigene Verarbeitungszentren. Wichtige europäische Produktionsgebiete, vor allem Rumänien, fielen als Lieferanten aus. Die Beschaffung der notwendigen Importe stieß auf zunehmende Schwierigkeiten, da solche aus Dollargebieten herrührten. Die USA. wiesen abnehmende exportable Überschüsse auf und betrieben eine auf lange Sicht gerichtete schonende Erdölpolitik. In diesem Sinne werden sogar Stimmen laut, welche die ständig wachsende karibische Produktion für den kommenden Importbedarf der USA. im Sinne einer «Hemisphärenwirtschaft» reservieren wollen. Gleichzeitig vird von den CEEC.-Ländern der Bedarf für 1947 auf 47453000 Tonnen und für 1951 auf 76763000 Tonnen geschätzt, wobei die Zunahme weniger auf Benzin als auf Betriebsöl entfällt. Es erhebt sich die Frage, aus welchem Gebiet dieser Importbedarf in Anbetracht der aufgedeckten Schwierigkeiten gedeckt werden kann.

In den dreißiger Jahren und während des Krieges sind nun im Mittleren Osten, und zwar in erster Linie im Bereich des Persischen Golfes, die wahrscheinlich bedeutendsten Erdöllager der Erde festgestellt worden. Mit etwa 3,7 Milliarden Tonnen (1948) übertreffen sie sogar die festgestellten Reserven der USA. Dagegen nahm die dortige Produktion erst nach dem Kriege in stärkerem Maße zu. Die mittlere Tagesproduktion lag 1938 bei 47000 Tonnen, 1945 bei 72000 Tonnen. 1946 jedoch schon bei 100000 Tonnen und im Sommer 1948 bei 118000 Tonnen. Damit wäre der Mittlere Osten in der Lage, den europäischen Bedarf weitgehend zu decken (Tagesbedarf für 1947 geschätzt auf 130000 Tonnen, 1951210000 Tonnen). Die Umstellung der Bedarfsdeckung aus dem Raume Golf-Karibisches Meer auf den Mittleren Osten begegnet jedoch weitgehenden Schwierigkeiten.

Die neuen Produktionsgebiete sind frachtungünstig gelegen; entweder muß die teure Suezkanalroute oder eine Pipeline quer durch Nordarabien gewählt werden. Die besondere Struktur des europäischen Bedarfes (Einfuhr von Erdölderivaten) bedingt die Errichtung von ganz bedeutenden neuen Verarbeitungszentren irgendwo zwischen Produktions- und Konsumptionsgebiet. Außerdem entfällt die Produktionssteigerung im Mittleren Osten auf Gesellschaften, die bisher an der europäischen Versorgung nicht beteiligt waren; es sind mit anderen Worten auch Umschichtungen in der Gesellschaftsstruktur notwendig.

Während des Krieges wurde der größte Teil der mittelöstlichen Produktion im Raume des Indik und in Südostasien verwendet; auch nach dem Kriege wurde die iranische Produktion fast ausschließlich in diesem Raume abgesetzt, wobei die Dollareinkünfte zur Deckung des britischen Importbedarfes aus dem amerikanischen Raume dienten. Die letzten Entwicklungen deuten jedoch darauf hin, daß gegenwärtig bewußt an einer Umstellung der europäischen Bedarfsdeckung auf den Mittleren Osten gearbeitet wird.

Neben einer Verdoppelung der bestehenden Pipelines nach Haifa und Tripoli bestehen zurzeit zwei weitere Gesellschaften, die Röhrenleitungen vom Persischen Golf an das Mittelmeer bauen wollen. Es scheint, daß die Endpunkte dieser Röhrenleitungen als Standorte der neuen Großraffinerien gewählt werden. Außerdem sind zwischen den produzierenden Gesellschaften und den den europäischen Markt beherrschenden Gesellschaften Abkommen getroffen worden, die einen weitgehenden Ausgleich schaffen. Schließlich betreffen einige weitere Abmachungen auch die Bezahlung, die anstatt in Dollars in englischen Pfund erfolgen kann. Auf eine detaillierte Darstellung der einzelnen Gesellschaften und ihre gegenseitigen Beziehungen wird an dieser Stelle verzichtet; es soll lediglich festgehalten werden, daß heute an Stelle der in der Vorkriegszeit wichtigsten Gesellschaften (Anglo Iranian Oil Co. und Iraq 